\title{
ON THE PROBLEM OF MODIFIED MOMENTS
}

\author{
RUPERT LASSER
}

\begin{abstract}
The problem of modified moments is studied. Let $\left(P_{n}(x)\right)_{n=0}^{\infty}$ be an orthogonal polynomial sequence. Given a sequence $\left(d_{n}\right)_{n=0}^{\infty}$ of real numbers, does there exist a bounded nondecreasing function with infinitely many points of increase such that for every $n \in \mathbf{N}_{0}, d_{n}=\int_{-\infty}^{\infty} P_{n}(x) d \mu(x)$ ? Is there any information about the support of $\mu$ ? A necessary and sufficient condition for the existence of such a function $\mu$ is given in terms of the positivity of certain determinants. For certain $\left(P_{n}(x)\right)_{n=0}^{\infty}$ a description of the support of $\mu$ is established.
\end{abstract}

Let $\left(P_{n}(x)\right)_{n=0}^{\infty}$ be an orthogonal polynomial sequence. A sequence $\left(d_{n}\right)_{n=0}^{\infty}$ of real numbers is called a sequence of modified moments with respect to $\left(P_{n}(x)\right)_{n=0}^{\infty}$ if there exists a bounded nondecreasing function $\mu$ with infinitely many points of increase such that

$$
d_{n}=\int_{-\infty}^{\infty} P_{n}(x) d \mu(x), \quad n=0,1,2, \ldots
$$

Modified moments are studied, e.g., in numerical analysis; see [2, p. 47].

Write the linearization of the product of any two orthogonal polynomials $P_{m}(x)$ and $P_{n}(x)$ :

$$
P_{m}(x) P_{n}(x)=\sum_{k=0}^{2 \min (m, n)} g(m, n, m+n-k) P_{m+n-k}(x) .
$$

Given $\left(d_{n}\right)_{n=0}^{\infty}$ denote

$$
d_{n m}=d_{m n}=\sum_{k=0}^{2 \min (m, n)} g(m, n, m+n-k) d_{m+n-k} .
$$

The classical theory of moments yields the following solution of the problem of modified moments.

THEOREM 1. Let $\left(P_{n}(x)\right)_{n=0}^{\infty}$ be an orthogonal polynomial sequence. Given a realvalued sequence $\left(d_{n}\right)_{n=0}^{\infty}$, the following are equivalent:

(a) $d_{n}=\int_{-\infty}^{\infty} P_{n}(x) d \mu(x)$, where $\mu$ is a nondecreasing function with infinitely many points of increase.

(b) $\operatorname{det}\left(\left(d_{i j}\right)_{0 \leqslant i, j \leqslant n}\right)>0$ for $n=0,1,2, \ldots$.

Received by the editors March 1, 1983.

1980 Mathematics Subject Classification. Primary 30E05, 33A65; Secondary 42C05.

Key words and phrases. Moment problems, orthogonal polynomials. 
Proof. Assume at first that (b) is valid. Define a functional $\psi$ on the space of all polynomials by

$$
\psi(p)=\sum_{n=0}^{N} a_{n} d_{n},
$$

provided $p(x)=\sum_{n=0}^{N} a_{n} P_{n}(x)$. By (b) we have $\psi\left(p^{2}\right)>0$ for each $p \neq 0$. Now [6, Chapter VI, problem 44] and Theorem 1.1 of [7] imply there exists a bounded nondecreasing function $\mu$ such that $\int_{-\infty}^{\infty} P_{n}(x) d \mu(x)=d_{n}$. The function $\mu$ has infinitely many points of increase. In fact, assume supp $\mu=\left\{x_{1}, \ldots, x_{n}\right\}$. Consider a polynomial $p \neq 0$ such that $p\left(x_{i}\right)=0$ for $i=1, \ldots, n$. Then

$$
p(x)=\sum_{n=0}^{N} a_{n} P_{n}(x)
$$

and

$$
\sum_{n, m=0}^{N} a_{n} a_{m} d_{n m}=\int_{-\infty}^{\infty} p^{2}(x) d \mu(x)=0,
$$

a contradiction to $(b)$.

Having more information about $P_{n}(x)$ one might ask for a stronger result. Assume the orthogonal polynomials $P_{n}(x)$ are random walk polynomials, that is

$$
\begin{gathered}
P_{0}(x)=1, \quad a_{0} P_{1}(x)=x-b_{0}, \\
a_{n} P_{n+1}(x)=P_{1}(x) P_{n}(x)-b_{n} P_{n}(x)-c_{n} P_{n-1}(x),
\end{gathered}
$$

where $a_{0}>0, b_{0} \in \mathbf{R}$ with $a_{0}+b_{0}=1$ and $a_{n}>0, b_{n} \geqslant 0, c_{n}>0$ with $a_{n}+b_{n}+c_{n}$ $=1, n \in \mathbf{N}$. Moreover, assume each linearization coefficient $g(m, n, m+n-k)$ in (L) is nonnegative. Then we shall say $\left(P_{n}(x)\right)_{n=0}^{\infty}$ satisfies property (P). In [5] we have established that there is an intimate relation between hypergroup structures on $\mathbf{N}_{0}=\mathbf{N} \cup\{0\}$ and orthogonal polynomial sequences having the positivity property (P). Jacobi polynomials $P_{n}^{(\alpha, \beta)}(x)$, where $\alpha \geqslant \beta>-1, \alpha+\beta+1 \geqslant 0$, associated Legendre polynomials $L_{n}^{\nu}(x)$, where $\nu \geqslant 0$, or continuous $q$-ultraspherical polynomials $C_{n}(x ; \beta \mid q)$ where $-1<\beta<1,0<q<1$ belong to this class. Many other examples can be found in [5]. Given $\left(P_{n}(x)\right)_{n=0}^{\infty}$ satisfying property (P), the character space of the corresponding hypergroup $\mathbf{N}_{0}$ can be identified with

$$
D_{S}=\left\{x \in \mathbf{R}:\left(P_{n}(x)\right)_{n=0}^{\infty} \text { is bounded }\right\} .
$$

One knows that $D_{S}$ is compact and supp $\pi \subseteq D_{S} \subseteq\left[1-2 a_{0}, 1\right]$ where $\pi$ is the orthogonalization function. Each character on $\mathbf{N}_{0}$ is given by $\alpha_{x}: \mathbf{N}_{0} \rightarrow \mathbf{R}, \alpha_{x}(n)=$ $P_{n}(x)$, where $x \in D_{S}$. Compare [5].

THEOREM 2. Let $\left(P_{n}(x)\right)_{n=0}^{\infty}$ be an orthogonal polynomial sequence satisfying property (P). Given a bounded real-valued sequence $\left(d_{n}\right)_{n=0}^{\infty}$ the following are equivalent:

(a) $d_{n}=\int_{D_{S}} P_{n}(x) d \mu(x)$, where $\mu$ is a nondecreasing function with infinitely many points of increase in $D_{S}$.

(b) $\operatorname{det}\left(\left(d_{i j}\right)_{0 \leqslant i, j \leqslant n}\right)>0$ for $n=0,1,2, \ldots$ 
Proof. Using Theorem 12.3B of [3] condition (b) yields a nondecreasing function $\mu$ with all points of increase in $D_{S}$ such that $d_{n}=\int_{D_{S}} P_{n}(x) d \mu(x)$. The remaining assertions of Theorem 2 follow as in the proof of Theorem 1.

REMARKS. (a) I want to thank the referee for his useful comments. (b) In [4] a theory of moment problems for $T$-systems is developed a concept encompassing orthogonal polynomial sets. Further, in [1, No. 12] C. Berg considered a stronger version of positive definiteness with respect to certain polynomials, including the ultraspherical polynomials.

\section{REFERENCES}

1. C. Berg. Studies définies négatives et espaces de Dirichlet sur la sphère, Sém. Brelot-Choquet-Deny, Théorie du Potentiel, 13e année, 1969/70.

2. C. Brezinski, Padé type approximation and general orthogonal polynomials, Birkhäuser, Basel, 1980.

3. R. I. Jewett, Spaces with an abstract convolution of measures, Adv. in Math. 18 (1975), 1-101.

4. S. Karlin and W. J. Studden, Tchebyscheff systems: with applications in analysis and statistics, Wiley Interscience, New York, 1966.

5. R. Lasser, Orthogonal polynomials and hypergroups, Rend. Mat. (7) (to appear).

6. C. Pólya and G. Szegö, Aufgaben und Lehrsätze aus der Analysis, vol. II, Springer-Verlag, Berlin and New York, 1964.

7. J. A. Shohat and J. D. Tamarkin, The problem of moments, Math. Surveys, no. 1, Amer. Math. Soc., Providence, R.I., 1943; reprinted 1983.

8. G. Szegö, Orthogonal polynomials, Amer. Math. Soc. Colloq. Publ., vol. 23, Amer. Math. Soc., Providence, R.I., 1959.

Mathematisches Institut der Technischen Universitat MUnchen, D-8000 MUNChen 2, West GERMANY 